existed. We calculated that we would have had to enroll at least 1,200 HCWs in each group to have an $80 \%$ probability of detecting such a small difference. Because this is more HCWs than we have available at this medical center, we conclude that, for practical purposes, in a medical center of our size, the two methods of training are equivalent.

It is interesting to note that, of the 52 participants in Group A who had been fit tested as part of their training and who had passed, three failed when they were fit tested again as part of our study. This suggests that there is some variability in results of fit testing, especially because the same individuals were retested. It may be that some of the participants who failed our fit test would pass if they were tested again, but we did not test this hypothesis, rather, we provided additional training to the 22 fit-test failures before they were retested. The accuracy of fit testing as a measure of the HCW's ability to wear a respirator correctly needs to be validated.

We reviewed the cases of the 22 employees who failed the fit test, looking for features that might contribute to their failure. We found the professions of the employees who failed were similar to those who passed. We specifically noted that none of the HCWs who failed had worn a beard. In fact, only 1 of 179 participants had a beard, and he passed the fit test. The body features of those who failed and who passed were similar, with the exception of the one employee who had a thin habitus and small facial features that made the creation of a tight face seal difficult with the standard one-size respirator. Presumably, this situation will be remedied now that respirators of different sizes are offered.
Assuming that the two methods of training $\mathrm{HCWs}$ to wear a respirator were equivalent in efficacy, we estimate that the hospital could save approximately $\$ 19,000$ annually by switching to classroom instruction and eliminating the requirement for fit testing. However, despite evidence to support this, we were reluctant to stop fit testing, because OSHA regulations require hospitals issuing respirators to fit test and fit check their employees periodically. Therefore, we continue fit testing as required by law, but instead of one-on-one training, our industrial hygienist currently instructs employees in groups of 6 to 10 , followed by individual qualitative fit testing. This change has cut the training time previously required per employee in half, resulting in saving some, but not all, of the $\$ 19,000$ in potential savings described.

\section{REFERENCES}

1. Centers for Disease Control and Prevention. Draft guidelines for preventing the transmission of tuberculosis in health care facilities. 2nd ed. Notice of Comment Period. Federal Register, October 12, 1993;58(195):52810-52854

2. Occupational Safety and Health Administration. Code of Federal Regulations 1910.134: occupational safety and health standards, personal protective equipment, respiratory protection. In: Code of Federal Regulations. Washington, DC: Office of the Federal Register, October 8, 1993.

3. Centers for Disease Control and Prevention. Guidelines for preventing the transmission of Mycobacterium tuberculosis in Health-Care Facilities, 1994. MMWR 1994;(No. RR-13)43:1-132.

4. American National Standards Institute, 11 West 42nd St, New York, NY 10036, "Practices for Respiratory Protection," ANZI Z88.2, 1980.

5. Department of Health and Human Services, Public Health Service, Centers for Disease Control, Department of Labor. Respiratory protective devices; final rules and notice. Federal Register, June 8, 1995;60(110):30336-30402.

\title{
Community-Acquired and Nosocomial Bacteremia Trends
}

\section{Gina Pugliese, RN, MS Martin S. Favero, $\mathbf{P h D}$}

In a recent report, researchers at Emory University's Crawford Long Hospital described the trends in nosocomial and community-acquired Staphylococcus aureus bacteremia during two periods 10 years apart: 1980 to 1983 and 1990 to 1993 . This hospital-based observational study showed that the overall rate of bacteremia doubled during the second 4 year period, with the rate of nosocomial bacteremia increasing from 4.59 to 9.44 cases per 1,000 discharges and the rate of community-acquired bacteremia increasing from 6.2 to 13.46 cases per 1,000 discharges. For nosocomial and community-acquired
$S$ aureus bacteremia, the rates more than tripled, with increases of 0.75 to 2.80 cases per 1,000 discharges and from 0.84 to 2.43 cases per 1,000 discharges, respectively.

Intravascular devices were not implicated in any community-acquired bacteremia from 1980 to 1983 , but were associated with $22 \%$ of communityacquired bacteremia in 1990 to 1993 . Methicillin-resistant $S$ aureus (MRSA) was responsible for $32 \%$ of nosocomial $S$ aureus bacteremia and $18.5 \%$ of community-acquired $S$ aureus bacteremia from 1990 to 1993. Patients with community-acquired MRSA had regular contact with healthcare settings, such as patients who had been hospitalized recently or who were nursing home residents.
The authors note that devicerelated infection, the leading source of nosocomial $S$ aureus bacteremia, has emerged as an important source of community-acquired bacteremia in their hospital. In addition, MRSA bacteremia, once confined to the hospital setting, is an increasing problem in the nonhospital setting. The authors conclude that the shifting of care, including parenteral therapy, to the outpatient setting most likely accounts for these observations.

FROM: Steinberg JP, Clark CC, Hackman BO. Nosocomial and community-acquired Staphylococcus aureus bacteremia from 1980 to 1993: impact of intravascular devices and methicillin resistance. Clin Infect Dis 1996;23:255-259 